\title{
DESARROLLO DE UN EQUIPO DE FOTOTERAPIA PARA LA ICTERICIA NEONATAL, BASADO EN UN ALGORITMO PARA EL TRATAMIENTO Y UN MÉTODO PREDICTIVO PARA LA DETECCIÓN DEL NIVEL DE BILIRRUBINA
}

\author{
Pedro Morales Licla ${ }^{1}$ Erick Laura Bravo², Carmen Dávila Aliaga³
}

\begin{abstract}
RESUMEN
Desde que se demostró la eficacia de la fototerapia, se ha convertido en el principal método para la prevención y el tratamiento de la hiperbilirrubinemia del recién nacido con ictericia neonatal. Por ende, el objetivo de este proyecto es decrementar con herramientas de innovación el nivel anormal de bilirrubina en la sangre y así situarlo en niveles de bajo riesgo. Para ello, el diseño propuesto consta de una fuente de luz azul, que ofrece una distribución lumínica uniforme en toda la superficie del neonato; un sistema de control que garantiza el nivel de irradiancia constante a lo largo del tratamiento, un método predictivo no invasivo para determinar el nivel de bilirrubina en el neonato y una interfaz programable que permite sugerir el tipo tratamiento más efectivo. Se incluyen pruebas de riesgo eléctrico según el estándar IEC 60601-1 para equipamiento médico.
\end{abstract}

Palabras clave: Fototerapia; Hiperbilirrubinemia; Ictericia neonatal; Irradiancia; Método predictivo; Control en lazo cerrado; Curvas de nomograma (Fuente: DeCS BIREME).

\section{DEVELOPMENT OF A PHOTOTHERAPY EQUIPMENT FOR NEONATAL JAUNDICE, BASED ON AN ALGORITHM OF TREATMENT AND A PREDICTIVE METHOD FOR DETECTION OF THE BILIRUBIN LEVEL}

\begin{abstract}
Since the efficacy of phototherapy has been demonstrated, it has become the main method for the prevention and treatment of hyperbilirubinemia in the neonate with neonatal jaundice. Therefore, the objective of this project is to decrease the abnormal level of bilirubin in the blood with innovation tools and to place it at low risk levels. For this, the proposed design consists of a blue light source, which offers a uniform light distribution throughout the neonate's surface; A control system that ensures the level of constant irradiance throughout the treatment, a noninvasive predictive method to determine the level of bilirubin in the neonate and a programmable interface that allows to suggest the most effective type of treatment. Electrical hazards according to IEC 60601-1 standard for medical equipment are included.
\end{abstract}

Key words: Phototherapy; Hyperbilirrubinemia; Neonatal jaundice; Irradiance; Predictive method; Close loop control; Nomogram curves (Source:MeSH NLM).

\section{INTRODUCCIÓN}

La insuficiente disponibilidad de equipos de fototerapia, principalmente en establecimientos médicos del sector público, ocasiona en algunos casos, que no se atienda oportunamente la demanda de atenciones de recién nacidos con ictericia neonatal y por ende, solo se brinde asistencia a los recién nacidos más graves, postergando la atención de otros recién nacidos afectados con la misma enfermedad. Se estima que el $80 \%$ de los recién nacidos prematuros y el $60 \%$ de los recién nacidos a término ${ }^{1}$, están en riesgo de presentar un cuadro de ictericia neonatal, debido al incremento del nivel de bilirrubina sobretodo indirecta en la sangre (hiperbilirrubinemia); la etiología es múltiple, dentro de las que destacan, la causa hemolítica por incompatibilidad de factor como de grupo sanguíneo, que puede ser muy agresiva; como asociada a prematuridad, infecciones, asfixia, deshidratación, lactancia materna, etc. Inclusive puede ser de etiología multifactorial. Frente a esta patología la conducta a seguir dependerá de los valores de la bilirrubina, aun cuando se conoce la capacidad limitada del hígado en el neonato, más aún prematuro para metabolizar la bilirrubina. Una de las clasificaciones usadas para la toma de decisiones es la de la Academia Americana de Pediatría. En algunos casos la conducta será expectante, en otros se requiere el uso de fototerapia pero cuando esta es insuficiente es necesario realizar una exanguineotransfusión. La finalidad es prevenir las consecuencias graves que conlleva esta enfermedad sin tratamiento adecuado, como es el kernicterus, que trae consigo la pérdida de audición, pérdida del habla, problemas del sistema nervioso, daño cerebral grave y en algunos casos la muerte.

Investigador Instituto Nacional Materno Perinatal. Bachiller en Ingeniería electrónica. Universidad San Martín de Porres. Lima-Perú

Investigador Instituto Nacional Materno Perinatal. Bachiller en Ingeniería electrónica. Universidad San Martín de Porres. Lima-Perú.

Médico pediatra neonatóloga, docente de la Universidad Nacional Federico Villareal, Directora Ejecutiva de Investigación, Docencia y Atención en Neonatología del Instituto Nacional Materno Perinatal. Lima- Perú 
La fototerapia logra favorecer la degradación de la bilirrubina a través de la absorción de luz por la bilirrubina, lo cual conduce principalmente a dos tipos de alteraciones moleculares: configuración ( $\mathrm{Z}$ a $\mathrm{E}$ o cis a trans) e isomerizaciones estructurales (vide infra), de esta manera el fotoisómero ya no puede cruzar la barrera hematoencefálica e impregnarse en el cerebro generando complicaciones graves, además es mejor eliminada por vía renal.

Para ello se propone degradar el nivel de bilirrubina en un tiempo menor al habitual (comprendido por lo general entre 6-12 horas), optimizando el tratamiento y siguiendo las recomendaciones de la Academia Americana de Pediatría.

\section{MATERIALES Y MÉTODOS}

Se realizó la elaboración de un equipo biomédico para realizar fototerapia, para un tratamiento adecuado y efectivo. El desarrollo del equipo propuesto se basa en las recomendaciones de la Academia Americana de Pediatría $^{2}$, según se muestra en la figura 1 , además de sugerencias por parte del cuerpo médico del departamento de Neonatología del Instituto Nacional Materno Perinatal.

\section{A. Modelamiento y simulación para garantizar una distribución lumínica uniforme}

La Figura 2 describe la geometría de un led y una ubicación iluminada en la superficie, donde $r$ es la distancia entre el led y la iluminación local, la proyección de r sobre la superficie es d, y h denota la distancia vertical entre el led y la superficie. El ángulo

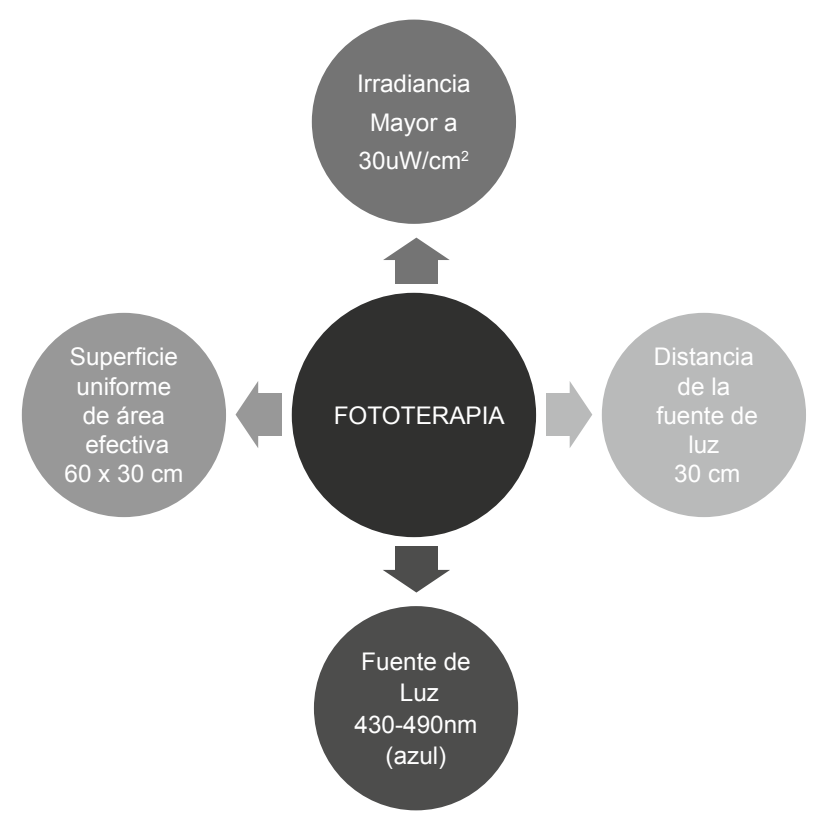

Figura 1. Recomendaciones de la Academia Americana de Pediatría. polar de la locación con respecto al led es denotado por $\theta$.

La potencia óptica por unidad de área está en función a d y h y viene dada por la siguiente ecuación (1) del modelo Lambertiano ${ }^{3}$.

$L(d, h)=\frac{(m+1) f_{0}}{2 \pi h^{2}}\left(1+\frac{d^{2}}{h^{2}}\right)^{-\frac{m+3}{2}}$

Donde $L(d, h)$ denota la intensidad lumínica en la unidad de lumen $/ \mathrm{m} 2$, f0 es el total de flujo luminoso (en lumenes) producido por el led, $\mathrm{m}$ es el número del modo Lambertiano y $m>0$. El número del modo es una medida de la directividad del haz de luz y se relaciona con el ángulo mitad del haz de luz a media potencia, denotado por $\Phi_{1 / 2}$.

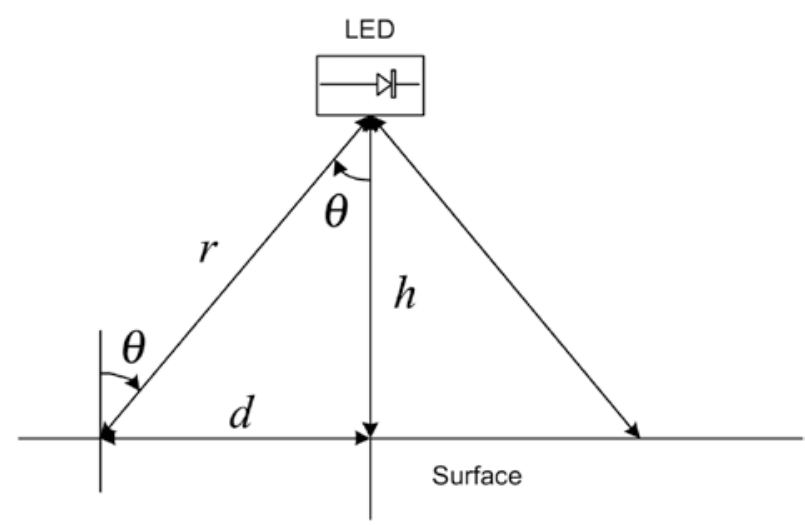

Figura 2. Geometría de un led y un punto de destino.

Los valores de iluminancia en lumen $/ \mathrm{m}^{2}$ de un led, para una superficie de $45 \times 45 \mathrm{~cm}$ a una altura de $30 \mathrm{~cm}$ con $\mathrm{f}_{0}=22, \mathrm{~m}=50$ y haciendo variar " $\mathrm{d}$ " de $5 \mathrm{~cm}$ en $5 \mathrm{~cm}$ se muestran en la siguiente tabla 01 .

Tabla 1. Distribución de un led en una superficie de $55 \times 45 \mathrm{~cm}$.

\begin{tabular}{|c|c|c|c|c|c|c|c|c|c|c|}
\hline 0 & 0 & 0 & 0 & 0.1 & 0.1 & 0.1 & 0 & 0 & 0 & 0 \\
\hline 0 & 0 & 0 & 0.5 & 3 & 5 & 3 & 0.5 & 0 & 0 & 0 \\
\hline 0 & 0 & 0.5 & 11 & 70 & 122 & 70 & 11 & 0.5 & 0 & 0 \\
\hline 0 & 0.1 & 3 & 70 & 487 & 960 & 487 & 70 & 3 & 0.1 & 0 \\
\hline 0 & 0.1 & 5 & 122 & 960 & 1984 & 960 & 122 & 5 & 0.1 & 0 \\
\hline 0 & 0.1 & 3 & 70 & 487 & 960 & 487 & 70 & 3 & 0.1 & 0 \\
\hline 0 & 0 & 0.5 & 11 & 70 & 122 & 70 & 11 & 0.5 & 0 & 0 \\
\hline 0 & 0 & 0 & 0.5 & 3 & 5 & 3 & 0.5 & 0 & 0 & 0 \\
\hline 0 & 0 & 0 & 0 & 0.1 & 0.1 & 0.1 & 0 & 0 & 0 & 0 \\
\hline
\end{tabular}

Los valores de cada celda de la tabla 01 especificadas en color mediante la simulación en Matlab, se muestran en la figura 3. 


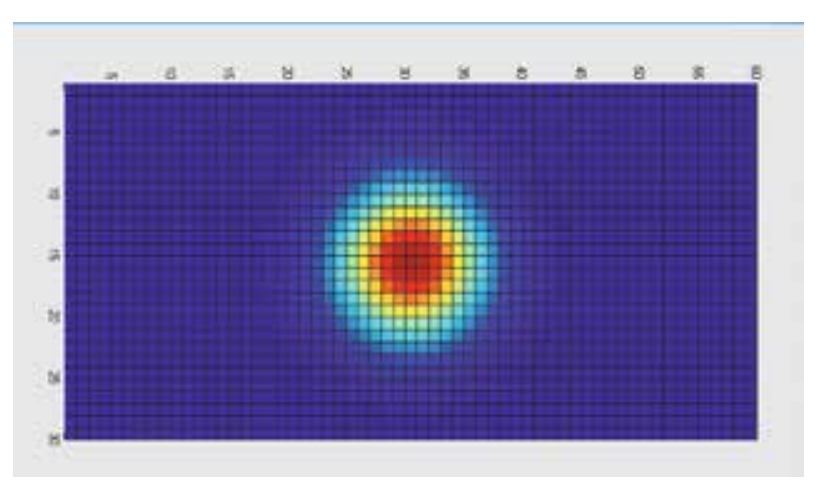

Figura 3. Distribución lumínica de un led en una superfcie de $60 \times 30 \mathrm{~cm}$.

La distribución lumínica uniforme en un área de 60×30 $\mathrm{cm}$, se logra superponiendo los modelos Lambertianos individuales de cada led distanciados entre sí a $8 \mathrm{~cm}$ desde sus centros. A continuación se muestra en la tabla 02 los valores de iluminancia para una matriz de leds de 7 filas y 4 columnas.

Tabla 2. Distribución de un led en una superficie de $35 \times 65 \mathrm{~cm}$.

\begin{tabular}{|c|c|c|c|c|c|c|}
\hline 582 & 904 & 957 & 943 & 957 & 904 & 582 \\
\hline 1718 & 2668 & 2822 & 2768 & 2822 & 2668 & 1718 \\
\hline 2046 & 3178 & 3362 & 3301 & 3362 & 3178 & 2046 \\
\hline 2121 & 3294 & 3485 & 3423 & 3485 & 3294 & 2121 \\
\hline 2105 & 3268 & 3458 & 3396 & 3458 & 3268 & 2105 \\
\hline 2087 & 3241 & 3429 & 3369 & 3429 & 3241 & 2087 \\
\hline 2130 & 3308 & 3499 & 3436 & 3499 & 3308 & 2130 \\
\hline 2087 & 3241 & 3429 & 3369 & 3429 & 3241 & 2087 \\
\hline 2105 & 3268 & 3458 & 3396 & 3458 & 3268 & 2105 \\
\hline 2121 & 3294 & 3485 & 3423 & 3485 & 3294 & 2121 \\
\hline 2046 & 3178 & 3362 & 3301 & 3362 & 3178 & 2046 \\
\hline 1718 & 2668 & 2822 & 2768 & 2822 & 2668 & 1718 \\
\hline 582 & 904 & 957 & 943 & 957 & 904 & 582 \\
\hline
\end{tabular}

Los valores de cada celda de la tabla 02 especificados en color mediante la simulación en Matlab, se muestran en la figura 4.

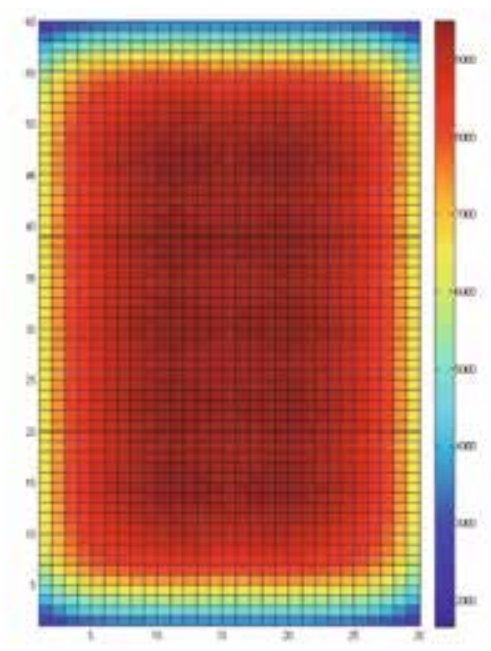

Figura 4. Distribución lumínica de una matriz de leds de 7 filas y 4 columnas.
B. Algoritmo de sugerencia de tipo de tratamiento según las curvas de nomograma

La National Institute for Health and Care Excellece (NICE), brinda herramientas como las curvas de nomograma, para sugerir un tipo tratamiento a la ictericia neonatal, basada en las semanas de gestación y el nivel de bilirrubina sérica total. Son 16 gráficos de umbral de tratamiento desde la semana 23 hasta la semana mayor o igual a $38^{4}$. Esta herramienta es de mucha utilidad, motivo por el cual ha sido implementada en nuestro proyecto siguiendo el diagrama de flujo mostrado en la figura 5.

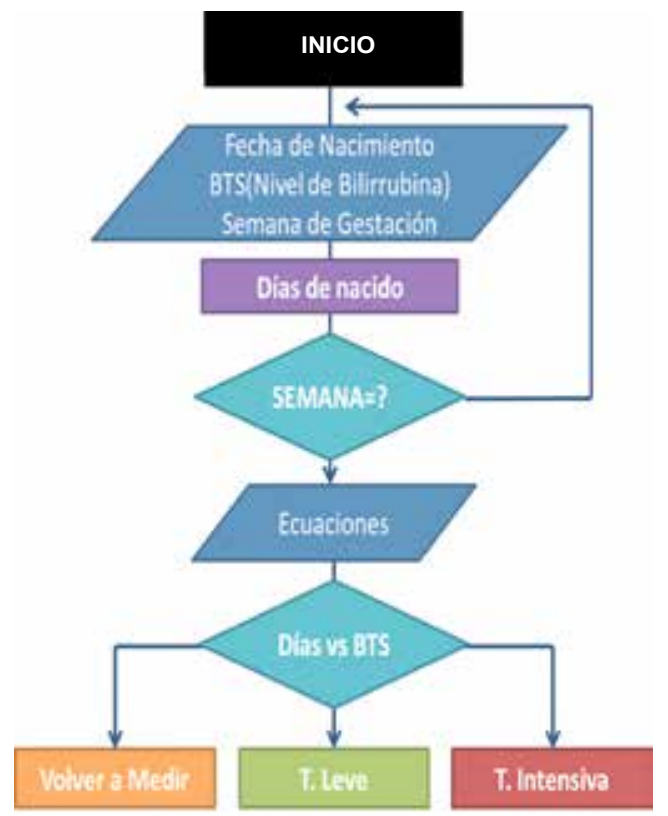

Figura 5. Diagrama de flujo del algoritmo de sugerencia de tipo de tratamiento según las curvas de nomograma.

\section{Método predictivo para la detección del nivel de bilirrubina}

Con el propósito de brindar un método de detección del nivel de bilirrubina adicional, se integró al equipo un método no invasivo basado en un modelo matemático - estadístico con un $78 \%$ de efectividad. ${ }^{5}$ Estas mediciones se hará continuamente a través del sistema de sensado y los valores serán actualizados en todo instante de tiempo en el sistema de control.

D. Control en lazo cerrado para el nivel de irradiancia Para garantizar el nivel de irradiancia mayor a 30uW/ $\mathrm{cm}^{2}$, umbral recomendado por la Academia Americana de Pediatría para un tratamiento efectivo, la irradiancia de la matriz de leds es controlado por un sistema de control en lazo cerrado tal como se muestra en la figura 5 , para que ante una posible descalibración por debajo del umbral del nivel de irradiancia, este se regule de forma autónoma. Para ello se utilizó el controlador PID; el cual, es uno de los controles más utilizados actualmente en la industria debido a su fácil sintonía y robustez en los diferentes procesos existentes ${ }^{3}$. 


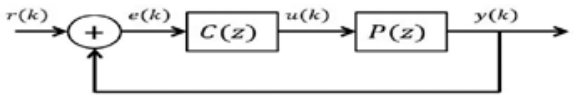

Figura 6. Diagrama de bloques del sistema de control

Donde:

$r(k)$ : Es señal de referencia o set point.

$e(k)$ : Es señal de error (resta entre $r(k)$ y $y(k))$.

$\mathrm{u}(\mathrm{k})$ : Es la señal de control (Ley de control).

$y(k)$ : Es nuestra variable de salida a controlar.

$C(z)$ : Es el controlador PID discreto.

$\mathrm{P}(\mathrm{z})$ : Es el proceso en representación discreta.

El método de sintonía utilizado es el Ziegler y Nichols que propone usar un periodo de muestreo menor $a \frac{\theta}{4}$. Para el control de irradiancia se escogió un periodo de muestreo de $T=0.1$, equivalente a $100 \mathrm{~ms}$ y a la vez menor $\mathrm{a} \frac{\theta}{4}$. Por ende el control discreto PDI viene dado por:

$$
\text { Donde: } \quad C\left(Z^{-1}\right)=\frac{u(k)}{e(k)}=\frac{q_{0}+q_{1} Z^{-1}+q_{2} z^{-2}}{1-Z^{-1}}
$$

$$
\begin{array}{r}
q_{0}=k_{p}\left[1+\frac{T}{2 t_{i}}+\frac{t_{d}}{T}\right] \\
q_{1}=-k_{p}\left[1-\frac{T}{2 t_{i}}+\frac{2 t_{d}}{T}\right] \\
q_{2}=\frac{k p * t_{d}}{T}
\end{array}
$$

Por ende de acuerdo a lo descrito la ley de control que se utilizará en el microcontrolador (ecuación 9) es la que se obtiene del control PID discreto, despejando $\mathrm{u}(\mathrm{k})$ y aplicando la transformada $\mathrm{Z}$.

$$
\begin{aligned}
& u(k)=u(k-1)+q_{0} e(k)+q_{1} e(k-1)+q_{2} e(k-2) \\
& \text { Donde: } \\
& \text { u(k): Es el control actual. } \\
& \mathrm{u}(\mathrm{k}-1) \text { : Control en un instante de muestreo atrás. } \\
& \mathrm{e}(\mathrm{k}) \text { : Es el error actual. } \\
& \mathrm{e}(\mathrm{k}-1) \text { : Error un instante de muestreo atrás. } \\
& \mathrm{e}(\mathrm{k}-2) \text { : Error dos instantes de muestreo atrás. }
\end{aligned}
$$

\section{E. Pruebas de seguridad eléctrica}

Se realizaron pruebas de riesgo eléctrico en el laboratorio de Biomédica de la PUCP, mediante un equipo de medición ESA 620, con fines de cumplir los estándares de la IEC 60601-1 para equipos médicos.

\section{RESULTADOS}

Se realizó el diseño y la elaboración de un equipo de fototerapia de bajo costo para el tratamiento de la hiperbilirrubinemia en recién nacidos que requieran este tipo de terapia de acuerdo a los niveles de bilirrubina presentados.
Se verifica que la representación de una matriz de leds es la superposición de todos los resultados de los modelos lambertianos individuales.

Validación del prototipo: En la presente etapa se evaluó la funcionalidad y desempeño del prototipo final analizando la repetitividad de sus funciones, grado del parámetro de irradiancia, homogenidad de luminosidad.

Para la ejecución del método predictivo se verificó una correcta medición de irradiancia en dos tiempos diferentes y de la misma forma un valor confiable de BTS inicial

El nivel de voltaje e intensidad lumínica medidas es inversamente proporcional al PWM que entrega la matriz de led, cumpliendo de esta forma la calibración autónoma (lazo cerrado).

Se realizó la evaluación de seguridad eléctrica para equipos de fototerapia.

\section{DISCUSIÓN}

La producción de equipos biomédicos en nuestro país es aún pobre, con serias limitaciones fundamentalmente presupuestales respecto a otros países.

Los equipos de fototerapia se usan en la atención neonatal desde más de 30 años; con el pasar de los años se han hecho presente tecnologías desde luz halógena hasta luz led. Sin embargo los principales equipos de fototerapia líderes que existen a nivel mundial dentro de sus principales funciones no poseen un medidor de irradiancia (uW/cm2) integrado, siendo este medido de forma externa a través de un radiómetro, si dichos niveles de irradiancia se encuentran fuera del umbral definido por cada terapia no hay ninguna calibración que garantice una terapia óptima.

Los principales equipos de fototerapia no monitorean de forma continua la degradación del nivel de bilirrubina y por ende no muestran en una pantalla el nivel final degradado, realizándolo de forma externa.

Finalmente la gran mayoría de equipos de fototerapia con excepción de la marca Alemana Draguer con su equipo nano blue, no almacenan reportes de las terapias aplicadas a los recién nacidos y por ende no son capaces de mantener en una base de datos una historia clínica por terapia realizada o en su defecto un muestreo en tiempo real para que el médico pueda visualizarlo.

La solución propuesta presenta alternativas de innovación adicionales a las funciones ya existentes en equipos de fototerapia. La inclusión de estas innovaciones fueron acogidas por necesidades de personal médico que por lo general operan equipos de fototerapia, con la finalidad de poder brindar una terapia más completa, óptima y eficiente al recién nacido. 
Por ende se propone un control en lazo cerrado de irradiancia integrado al equipo que se basa de un arreglo de sensores para medir el nivel de irradiancia de forma constante, un método predictivo no invasivo para la detección del nivel de bilirrubina y un sistema de reportes de los datos más importantes según la terapia aplicada.

Queda pendiente una segunda etapa en la que se evaluará la reproducibilidad de resultados del equipo en el tratamiento de recién nacidos con hiperbilirrubinemia. Este equipo biomédico ofrecerá una alternativa de bajo costo para el tratamiento de ictericia en la etapa neonatal. Demostrada su eficacia y seguridad en estudios posteriores, este equipo también permitirá reducir la tasa de discapacidad neonatal asociada a Kernicterus.

\section{AGRADECIMIENTOS}

Agradecimiento al Instituto Nacional Materno Perinatal por el apoyo a través de sus diferentes departamentos y servicios.

\section{REFERENCIAS BIBLIOGRÁFICAS}

1. Guía práctica clínica. Detección oportuna, diagnóstico y tratamiento de la hiperbilirrubinemia en niños mayores de 35 semanas de gestación hasta las 2 semanas de vida extrauterina. México: Secretaría de Salud, 2009.

2. Clinical practice guideline. Management of Hyperbilirubinemia in the Newborn Infant 35 or more weeks of gestation. USA: American Academy of Pediatrics, 2004.
3. Zhang G. Solid State Lighting Technology and Application Series. 1era edición. USA: Springer, 2012.

4. Nice clinical guideline. Neonatal Jaundice Treatment threshold graphs. UK: National Institute for Health and Clinical Excellence, 2010.

5. Osaku N, Silvério H. Phototherapy of the Newborn: a Predictive Model for the Outcome. Proc. IEEE, pp. 1-4. China, september 2005.

6. Mazzi E, Hiperbilirrubinemia Neonatal. Bolivia: Organización Panamericana de la Salud, 2005.

7. IES. The IESNA Lighting Handbook Reference \& Application. 9na edición. USA: IESNA, 2000.

8. Hunt R.W.G., Pointer M.R. Measuring Colour. 4ta edición. UK: The Wiley - Imaging Science and Technology, 2011.

9. Boyce P. Human Factors in Lighting, 2da edición. USA: Taylor \& Francis, 2003.

10. Koshel R. Illumination Enegineering. 1era edición. USA: IEEE y Wiley, 2013.

11. Ohta N, Robertson A. Colorimetry Fundamentals and Applications. 1era edición. UK: Wiley, 2005.

12. Rodriguez J, Figueras J. Ictericia Neonatal. ESPAÑA: Asociación española de pediatría, 2008.

13. International Commission on Illumination. Measurement of LEDs. 2da edición. Austria: CIE, 2007.

14. Zukauskas A, Shur M. Introduction to Solid-State Lighting. 1era edición. USA: Wiley, 2002.

15. Mottier P. LEDs for Lighting Applications. 1era edición. USA: Wiley, 2009.

16. Nice clinical guideline. Neonatal Jaundice Treatment threshold graphs. UK: National Institute for Health and Clinical Excellence, 2010.Lamola A. A Phamacologic view do Phototherapy. Clin Perinatol 43 (2016) 259-276

17. Lamola A. A Phamacologic view do Phototherapy. Clin Perinatol 43 (2016) 259-276

ORCID iDs

Carmen Dávila Aliaga $\quad$ (i) https://orcid.org/0000-0001-9411-5703 